\title{
A Mathematical Model for the Vector Transmission and Control of Banana Xanthomonas Wilt
}

\author{
Kweyunga Eliab Horub ${ }^{1}$, \& Tumwiine Julius ${ }^{2}$ \\ ${ }^{1}$ Department of Mathematics, Kabale University, Kabale, Uganda \\ ${ }^{2}$ Department of Mathematics, Mbarara University of Science and Technology, Mbarara, Uganda \\ Correspondence: Kweyunga Eliab Horub, Department of Mathematics, Kabale University, P. O. Box 317, Kabale, \\ Uganda. E-mail: hkweyunga@kab.ac.ug
}

Received: April 24, 2017 Accepted: May 16, 2017 Online Published: July 19, 2017

doi:10.5539/jmr.v9n4p101～URL: https://doi.org/10.5539/jmr.v9n4p101

\begin{abstract}
Banana Xanthomonas wilt is currently wrecking havoc in East and Central Africa. In this paper, a novel theoretical model for the transmission of banana Xanthomonas wilt by insect vectors is formulated and analyzed. The model incorporates roguing of infected plants and replanting using healthy suckers. The model is analyzed for the existence and stability of the equilibrium points. The global stability of the disease-free equilibrium point was determined by using a Lyapunov function and LaSalle's invariance principle. For the global stability of the endemic equilibrium point, the theory of competitive systems, compound matrices and stability of periodic orbits were used. It was established that if the basic reproduction number satisfies $R_{0} \leq 1$, the disease-free equilibrium point is globally stable and the disease will be wiped out and if $R_{0}>1$, the endemic equilibrium is stable and the disease persists. A numerical simulation of the model was also carried out. It was found out that at appropriate roguing and replanting, the disease can be contained.
\end{abstract}

Keywords: Banana Xanthomonas wilt, basic reproduction number, competitive systems, disease control, endemic equilibrium, roguing

\section{Introduction}

Banana is the world's fourth most important food crop after rice, wheat and maize. Approximately one-third of bananas produced globally are grown in sub-Saharan Africa. The great lakes region of Africa is the largest banana producing and consuming region in Africa with Uganda the second largest producer after India. Being a perennial crop with all year-round fruiting and harvesting abilities, banana plays a significant role as it represents a source of food and income for most Ugandans. It's estimated that banana production contributes up to $22 \%$ of national agricultural rural income in Uganda (Tripathi et al, 2008).

However the production of bananas in Uganda is threatened by the outbreak of Banana Xanthomonas wilt caused by Xanthomonas campestris pv. musacearum. The disease is mainly transmitted by insect vectors through the male buds (Tushemereirwe et al, 2004) and Rutikanga et al., 2016). Contaminated farming tools, infected planting materials, nectarsucking birds and bats are the other means of transmission of the bacterium (Tinzaara et al., 2006; Buregyeya et al., 2014). Insects pick up the bacterium externally on their body parts when they visit the banana inflorescence and transmit it to healthy plants while foraging for pollen and juice (Tinzaara et al., 2006). It is characterized by progressive yellowing and wilting of leaves, uneven and premature ripening of fruit, wilting of bracts, shriveling and rotting of male buds and a characteristic yellow ooze from the cut pseudo stem (Eden-Green, 2004; Tushemereirwe et al, 2004).

Management measures include roguing of diseased banana plants, debudding or removal of the male buds using a forked stick, use of clean planting materials and sterilization of farm tools using chemicals or a flame of fire (Tinzaara et al., 2016; Kubiriba et al., 2014; Kubiriba \& Tushemereirwe, 2014; Jogo et al., 2013). In the absence of a cure (chemical or biological) and unavailability of resistant varieties, the disease continues to spread to new areas. It should however be noted that where these management options have been strictly applied, the disease has been contained .

Mathematical models for plant epidemics have been developed with the ultimate purposes of developing control strategies and assessing their effectiveness. Such models have been developed by Fishman et al. (1983), Fishman \& Marcus (1984), Chan \& Jeger (1994), Holt et al. (1997), Holt et al.(1999), Madden et al. (2000), Gibson et al. (2004), Jeger et al. (2004), Tang et al. (2010), Zhonghua \& Yaohong (2014), Nannyonga et al. (2015) and Nakakawa et al.(2016) among others. Holt et al. (1999) formulated an epidemiological model for identifying control strategies for tomato leaf curl virus disease in Southern India. Chan \& Jeger (1994) developed an analytical model of plant virus disease dynamics with roguing and replanting of healthy plants and assessed the effectiveness of roguing. Fishman \& Marcus (1984) formulated 
and analyzed a model for the spread of a plant disease with periodic removal of infected plants while Gibson et al.(2004) developed a model to study the control strategies for sweet potato virus disease in Africa. Furthermore, epidemiological and economic models were formulated to study the spread and control of citrus tristeza virus disease (Fishman et al., 1983); the models were used to assess the effectiveness of the eradication policy. An epidemiological model incorporating vector dynamics applied to African Cassava Mosaic virus disease was considered by Holt et al. (1997) who concluded that roguing of diseased plants had little effect on disease incidence. Jeger et al. (2004) modeled disease dynamics and control interventions for insect transmitted plant epidemics while Nannyonga et al.(2015) applied optimal control theory to study the vector and contaminated tool preventive methods to control the spread of banana Xanthomonas wilt within plantations. Clearly, literature is abundant on modeling the dynamics of many plant epidemics with the aim of gaining insights on epidemic development and control. Most of these models incorporate replanting and roguing of infected plants, see Tang et al. (2010) and Zhonghua \& Yaohong (2014) and the references cited there in. However, models for the dynamics of banana Xanthomonas wilt transmitted by insect vectors are scarce in literature. The model proposed in this study also incorporates the roguing of infected banana plants and replanting using healthy suckers. The effectiveness and suitability of these measures is assessed.

This paper is organized as follows: In Section 2, we formulate the model, determine the equilibrium points and analyze their stability. In Section 3, a numerical simulation of the model is carried out while in Section 4, we discuss the results and make recommendations.

\section{Model Formulation and Analysis}

We assume that there is no latency in both the host and vector populations and that the transmission of the bacterium by the vectors is by non-circulative non-persistent mode. We also assume that the emigration and immigration rates of the vectors are equal so that the total vector population size is constant. It is further assumed that the roguing rate of infected plants is balanced by the replanting rate by the healthy suckers. Banana xanthomonas wilt is a systematic disease in that the whole plant is infected and can therefore be used as a conceptual unit of modeling. The total banana plant population size at time $t$ given by $N_{H}(t)$, is partitioned into subclasses of healthy and infected plants with sizes denoted by $S_{H}(t)$ and $I_{H}(t)$, respectively. Recruitment into the healthy plant population is via two processes namely, emergency of new suckers at a constant rate $\lambda_{1}$ and replanting using healthy suckers. Healthy plants are harvested at a rate $\mu$ whose reciprocal is the life time of a healthy banana plant. Roguing of infected plants is done at a rate $r$ which is also the replanting rate of healthy suckers. The death rate of infected plants is assumed to be at a constant rate $d$. The vector population is divided into two classes; the susceptible and infective vectors whose sizes at time $t$ are $S_{V}(t)$ and $I_{V}(t)$ respectively. Recruitment into the susceptible vector population is through the immigration of vectors which depends on the total vector population size $N_{V}(t)$. The emigration rate of both classes of vectors is assumed to be equal to the immigration rate at a constant $g$.

Healthy banana plants become infected through contact with infective vectors through a standard incidence term $\beta_{1} S_{H} \frac{I_{V}}{N_{H}}$ where $\beta_{1}$ is the contact rate between infective vectors and healthy banana plants. Similarly, susceptible vectors acquire the bacterium through contact with infected banana plants through a standard incidence term $\beta_{2} S_{V} \frac{I_{H}}{N_{H}}$ where $\beta_{2}$ is the contact rate between infected banana plants and non-infective vectors and $\beta_{2} \frac{I_{H}}{N_{H}}$ is the force of infection.

The following system of ordinary differential equations based on the basic model assumptions, parameters and variables is derived to describe the dynamics of the vector transmission of banana Xanthomonas wilt and it's control:

$$
\begin{aligned}
\frac{d S_{H}}{d t} & =\left(\lambda_{1}+r-\mu\right) S_{H}-\beta_{1} S_{H} \frac{I_{V}}{N_{H}}, \\
\frac{d I_{H}}{d t} & =\beta_{1} S_{H} \frac{I_{V}}{N_{H}}-(r+d) I_{H}, \\
\frac{d S_{V}}{d t} & =g N_{V}-\beta_{2} S_{V} \frac{I_{H}}{N_{H}}-g S_{V}, \\
\frac{d I_{V}}{d t} & =\beta_{2} S_{V} \frac{I_{H}}{N_{H}}-g I_{V},
\end{aligned}
$$

where $N_{H}=S_{H}+I_{H}$ is the total host plant population and satisfies the equation $\frac{d N_{H}}{d t}=\left(\lambda_{1}+r-\mu\right) S_{H}-(r+d) I_{H}$ and $N_{V}=S_{V}+I_{V}$ is the total vector population and satisfies the equation $\frac{d N_{V}}{d t}=0$. It is noted that the total banana plant population size is variable whereas the total vector population size is constant. In the absence of the disease, the entire banana plantation consists of only the healthy plants and $r=0$. The total population of the healthy plants grows exponentially when $\lambda_{1}>\mu$, declines exponentially to extinction when $\lambda_{1}<\mu$, and is constant for $\lambda_{1}=\mu$. In this study we assume that $\lambda_{1}>\mu$.

For convenience, we carry out a non-dimensionalization of the model as follows: Define $s_{h}=\frac{S_{H}}{N_{H}}, i_{h}=\frac{I_{H}}{N_{H}}, s_{v}=\frac{S_{V}}{N_{V}}$, 
$i_{v}=\frac{I_{V}}{N_{V}}$ and $m=\frac{N_{V}}{N_{H}}$. By differentiating with respect to time and simplifying, we obtain the following system of equations:

$$
\begin{aligned}
\frac{d s_{h}}{d t} & =\alpha s_{h}-\beta_{1} m s_{h} i_{v}-\alpha s_{h}^{2}+\phi s_{h} i_{h}, \\
\frac{d i_{h}}{d t} & =\beta_{1} m s_{h} i_{v}-\alpha s_{h} i_{h}-\phi i_{h}+\phi i_{h}^{2}, \\
\frac{d s_{v}}{d t} & =g-\beta_{2} s_{v} i_{h}-g s_{v}, \\
\frac{d i_{v}}{d t} & =\beta_{2} s_{v} i_{h}-g i_{v},
\end{aligned}
$$

where $\alpha=\lambda_{1}+r-\mu$ and $\phi=r+d$. Further, using the substitution $s_{v}=1-i_{v}$, system (2) can be reduced to the following system of three ordinary differential equations:

$$
\begin{aligned}
\frac{d s_{h}}{d t} & =\alpha s_{h}\left(1-s_{h}\right)-\beta_{1} m s_{h} i_{v}+\phi s_{h} i_{h}, \\
\frac{d i_{h}}{d t} & =\beta_{1} m s_{h} i_{v}-\alpha s_{h} i_{h}-\phi i_{h}\left(1-i_{h}\right), \\
\frac{d i_{v}}{d t} & =\beta_{2} i_{h}\left(1-i_{v}\right)-g i_{v} .
\end{aligned}
$$

For biological reasons, the model is analyzed in the feasible region

$\Gamma=\left\{\left(s_{h}, i_{h}, i_{v}\right) \in \mathfrak{R}_{+}^{3} \mid s_{h}, i_{h}, i_{v} \geq 0, s_{h}+i_{h}=1,0 \leq i_{v} \leq 1\right\}$,

where $\Gamma$ is positively invariant with respect to system (3) and $\mathfrak{R}_{+}^{3}$ denotes a nonnegative cone of $\mathfrak{R}^{3}$ including its lower dimensional faces. Denote by $\partial \Gamma$ and $\dot{\Gamma}$ the boundary and interior of $\Gamma$ in $\mathfrak{R}^{3}$ respectively.

\subsection{Equilibrium Points and Basic Reproduction Number}

To determine the equilibrium points, the right hand-side of system (3) is equated to zero. Calculations show that system (3) has four equilibrium points namely $E_{0}(0,0,0), E_{1}(1,0,0), E_{2}\left(0,1, \frac{\beta_{2}}{\beta_{2}+g}\right)$ and $E_{3}\left(s_{h}^{*}, i_{v}^{*}, i_{h}^{*}\right)$ where $s_{h}^{*}=\frac{(\alpha+\phi)\left(\beta_{2}+g\right)-\beta_{1} \beta_{2} m}{\beta_{2}(\alpha+\phi)}$, $i_{h}^{*}=\frac{\beta_{1} \beta_{2} m-g(\alpha+\phi)}{\beta_{2}(\alpha+\phi)}$ and $i_{v}^{*}=\frac{\beta_{1} \beta_{2} m-g(\alpha+\phi)}{\beta_{1} \beta_{2} m}$. In this study, the two equilibrium points of interest are $E_{1}$, the disease free equilibrium and $E_{3}$, the endemic equilibrium point.

The basic reproduction number, $R_{0}$, defined by Anderson \& May (1991) as the number of secondary infectives arising out of one infective introduced in the disease free population, is determined using the approach by van den Driessche \& Watmough (2002) and found to be given by the expression $R_{0}=\sqrt{\frac{\beta_{1} \beta_{2} m}{g\left(\lambda_{1}+2 r+d-\mu\right)}}$.

\subsection{Local Stability of Disease-free Equilibrium Point}

The Jacobian matrix evaluated at the disease-free equilibrium point, $E_{1}=(1,0,0)$, is

$$
J_{E_{1}}=\left(\begin{array}{ccc}
-\alpha & \phi & -\beta_{1} m \\
0 & -(\alpha+\phi) & \beta_{1} m \\
0 & \beta_{2} & -g
\end{array}\right),
$$

which has three eigenvalues one being $-\alpha$ and the other two are obtained from the sub-matrix

$$
J_{E_{1}^{\prime}}=\left(\begin{array}{cc}
-(\alpha+\phi) & \beta_{1} m \\
\beta_{2} & -g
\end{array}\right),
$$

whose trace $\left(J_{E_{1}^{\prime}}\right)=-(\alpha+\phi+g)$ and determinant $\left(J_{\left(E_{1}^{\prime}\right)}\right)=(\alpha+\phi) g-\beta_{1} \beta_{2} m$. For the disease free equilibrium point to be locally asymptotically stable, all the eigenvalues must have negative real parts. In this case, the trace $\left(J_{E_{1}^{\prime}}\right)$ is negative while the determinant should be positive. Clearly trace $<0$ and determinant $>0$ if $R_{0}<1$. Thus, the disease free equilibrium point is locally asymptotically stable if $R_{0}<1$.

\subsection{Global Stability of Disease Free Equilibrium Point}

The global stability of the disease-free equilibrium point is determined using Lyapunov's direct method (also called the second method of Lyapunov) as follows: Consider a dependent variable $L=g i_{h}+\beta_{1} m i_{v}$ which is such that

1. $L \geq 0$ along the solutions of system (3) 
2. $L=0$ if and only if $i_{h}=0$ and $i_{v}=0$

The derivative of $L$ along the solutions of system (3) is

$$
\begin{aligned}
L^{\prime} & =g \beta_{1} m s_{h} i_{v}-(\alpha+\phi) g s_{h} i_{h}+\beta_{1} \beta_{2} m i_{h}\left(1-i_{v}\right)-g \beta_{1} m i_{v}, \\
& \leq\left[-(\alpha+\phi) g s_{h} i_{h}+\beta_{1} \beta_{2} m i_{h}\left(1-i_{v}\right)\right], \\
& =(\alpha+\phi) g i_{h}\left[\frac{\beta_{1} \beta_{2} m\left(1-i_{v}\right)}{(\alpha+\phi) g}-s_{h}\right], \\
& =\left[R_{0}^{2}\left(1-i_{v}\right)-s_{h}\right](\alpha+\phi) g i_{h},
\end{aligned}
$$

since $\beta_{1} \beta_{2} m=(\alpha+\phi) g R_{0}^{2}$.

In the first inequality, the term $g \beta_{1} m s_{h} i_{v}-\beta_{1} m g i_{v}<0$ in $\Gamma$ and has been neglected.

In the case that $R_{0}<1$, then $L^{\prime}=0$ if and only if $i_{h}=0$. For the case that $R_{0}=1$, then $L^{\prime}=0$ if and only if $i_{h}=0$, $i_{v}=0$ and $s_{h}=1$. For the case when $R_{0}>1$, then $L^{\prime}>0$ for $s_{h}$ sufficiently close to 1 except when $i_{h}=i_{v}=0$. Solutions starting sufficiently close to $E_{1}$ leave a neighborhood $E_{1}$ except those on the invariant $s_{h}$-axis, on which system (3) reduces to $s_{h}^{\prime}=\alpha s_{h}\left(1-s_{h}\right)$ and thus $s_{h} \longrightarrow 1$ as $t \longrightarrow \infty$. In all cases, the largest compact invariance set in $\left\{\left(s_{h}, i_{h}, i_{v}\right) \in \Gamma: L^{\prime}=0\right\}$ when $R_{0} \leq 1$ is the singleton $\left\{E_{1}\right\}$. LaSalle's invariance principle (LaSalle, 1976) then implies that $E_{1}$ is globally asymptotically stable in $\Gamma$.

\subsection{Local Stability of the Endemic Equilibrium Point}

For simplification, the coordinates of the endemic equilibrium point can be expressed in terms of the basic reproduction number $R_{0}$ as $s_{h}^{*}=1-\frac{g}{\beta_{2}}\left(R_{0}^{2}-1\right), i_{h}^{*}=\frac{g}{\beta_{2}}\left(R_{0}^{2}-1\right)$ and $i_{v}^{*}=\frac{R_{0}^{2}-1}{R_{0}^{2}}$. Clearly, the endemic equilibrium point $E_{3}$ exists only when $R_{0}>1$ otherwise it will not make biological sense.

The Jacobian matrix evaluated at the endemic equilibrium point is

$$
J_{E_{3}}=\left(\begin{array}{ccc}
-\alpha s_{h}^{*} & \phi s_{h}^{*} & -\beta_{1} m s_{h}^{*} \\
\phi i_{h}^{*} & -\alpha s_{h}^{*}-\phi\left(1-2 i_{h}^{*}\right) & \beta_{1} m s_{h}^{*} \\
0 & \frac{g i_{v}^{*}}{i_{h}^{*}} & -g R_{0}^{2}
\end{array}\right) .
$$

Let $p=\frac{g}{\beta_{2}}\left(R_{0}^{2}-1\right)=i_{h}^{*}$, then system (5) can be simplified to

$$
J_{E_{3}}=\left(\begin{array}{ccc}
-\alpha(1-p) & \phi(1-p) & -\beta_{1} m(1-p) \\
\phi p & -\alpha(1-p)-\phi(1-2 p) & \beta_{1} m(1-p) \\
0 & \frac{\beta_{2}}{R_{0}^{2}} & -g R_{0}^{2}
\end{array}\right) .
$$

The characteristic equation is obtained from $\operatorname{det}\left(J_{E_{3}}-\lambda I\right)=0$, where $\lambda$ represents the eigenvalues and $I$ is the identity matrix and is given by

$$
\begin{gathered}
\lambda^{3}+\lambda^{2}\left[g R_{0}^{2}+2 \alpha(1-p)+\phi(1-2 p)\right]+\lambda\left[g R_{0}^{2}\{\alpha(1-p)+\phi(1-2 p)\}-g(\alpha+\phi)(1-p)\right. \\
+\alpha(1-p)\left\{g R_{0}^{2}+\alpha\left((1-p)+\phi(1-2 p)-\phi^{2} p(1-p)\right]\right. \\
+g R_{0}^{2} \alpha(1-p)[\alpha(1-p)+\phi(1-2 p)]-\alpha g(\alpha+\phi)(1-p)^{2}+(\alpha+\phi) g p \phi(1-p)-g r_{0}^{2} p \phi(1-p)=0,
\end{gathered}
$$

which is of the form

$$
\lambda^{3}+a_{1} \lambda^{2}+a_{2} \lambda+a_{3}=0
$$

where

$$
\begin{gathered}
a_{1}=g R_{0}^{2}+\alpha(1-p)+\phi(1-2 p)+\alpha(1-p), \\
=g R_{0}^{2}+(2 \alpha+\phi)(1-p)-\phi p . \\
a_{2}=g R_{0}^{2}[\alpha(1-p)+\phi(1-2 p)]-g(\alpha+\phi)(1-p)+\alpha(1-p)\left[g R_{0}^{2}+\alpha(1-p)+\phi(1-2 p)\right]-\phi^{2} p(1-p), \\
a_{2}=g R_{0}^{2}[(\alpha+\phi)(1-p)+\alpha(1-p)-p \phi]+(\alpha+\phi)(1-p)[\alpha(1-p)-g-p \phi] .
\end{gathered}
$$


and the constant term $a_{3}$ is given by

$$
\begin{aligned}
a_{3} & =g R_{0}^{2} \alpha(1-p)[\alpha(1-p)+\phi(1-2 p)]-\alpha g(\alpha+\phi)(1-p)^{2}+(\alpha+\phi) g p \phi(1-p)-g R_{0}^{2} \phi^{2} p(1-p), \\
& =g(1-p)(\alpha+\phi)(\alpha-p(\alpha+\phi))\left[R_{0}^{2}-1\right] .
\end{aligned}
$$

By the Routh-Hurwitz criteria, the endemic equilibrium point is locally asymptotically stable if $a_{1}, a_{2}, a_{3}>0$ and $a_{1} a_{2}>$ $a_{3}$. Now, $a_{1}>0, a_{2}>0$ and $a_{3}>0$ if $\alpha>p(\alpha+\phi)$ and $R_{0}>1$.

It remains to show whether $a_{1} a_{2}>a_{3}$. Now,

$$
\begin{gathered}
a_{1} a_{2}-a_{3}=\left[g R_{0}^{2}+(\alpha+\phi)(1-p)+\alpha-p(\alpha+\phi)\right]\left[g r_{0}^{2}\{(\alpha+\phi)(1-p)+\alpha-p(\alpha+\phi)\}+(\alpha+\phi)(1-p)\{\alpha-p(\alpha+\phi)\}\right] \\
-g R_{0}^{2}(1-p)(\alpha+\phi)\{\alpha-p(\alpha+\phi)\}+g(\alpha+\phi)(1-p)\{\alpha-p(\alpha+\phi)\} \\
=\left(g R_{0}^{2}\right)^{2}[(\alpha+\phi)(1-p)+\alpha-p(\alpha+\phi)]+(\alpha+\phi)(1-p) g R_{0}^{2}[\alpha-p(\alpha+\phi)] \\
+g R_{0}^{2}(\alpha+\phi)(1-p)[(\alpha+\phi)(1-p)+\alpha-p(\alpha+\phi)] \\
+(\alpha+\phi)^{2}(1-p)^{2}[\alpha-p(\alpha+\phi)]+g R_{0}^{2}(\alpha-p(\alpha+\phi))[(\alpha+\phi)(1-p)+\alpha-p(\alpha+\phi)] \\
+(\alpha-p(\alpha+\phi))(\alpha+\phi)(1-p)[\alpha-p(\alpha+\phi)]-g R_{0}^{2}(1-p)(\alpha+\phi)[\alpha-p(\alpha+\phi)] \\
+g(\alpha+\phi)(1-p)[\alpha-p(\alpha+\phi)] .
\end{gathered}
$$

Thus,

$$
\begin{gathered}
a_{1} a_{2}-a_{3}=\left(g R_{0}^{2}\right)^{2}[(\alpha+\phi)(1-p)+\alpha-p(\alpha+\phi)]+g R_{0}^{2}(\alpha+\phi)(1-p)[(1-p)(\alpha+\phi)+\alpha-p(\alpha+\phi)] \\
+(\alpha+\phi)^{2}(1-p)^{2}[\alpha-p(\alpha+)]+g R_{0}^{2}(\alpha-P(\alpha+\phi))[(\alpha+\phi)(1-p)+\alpha-p(\alpha+\phi)] \\
(\alpha+\phi)(1-p)(\alpha-p(\alpha+\phi))[\alpha-p(\alpha+\phi)-g]+ \\
g(\alpha+\phi)(1-p)[\alpha-p(\alpha+\phi)]-g^{2} R_{0}^{2}(\alpha+\phi)(1-p) .
\end{gathered}
$$

It is clear that $a_{1} a_{2}>a_{3}$ when $\alpha>p(\alpha+\phi)$.

Since $a_{1}, a_{2}, a_{3}>0$ and $a_{1} a_{2}>a_{3}$, then by the Routh-Hurwitz criteria for the characteristic polynomial of degree 3 , the endemic equilibrium point is locally asymptotically stable when $R_{0}>1$.

\subsection{Global Stability of the Endemic Equilibrium Point}

The global stability of the endemic equilibrium point is determined using the approach developed by Li \& Muldowney (1995) for SEIR models. We start with the preliminaries.

Let $x \mapsto f(x)$ be a $C^{\prime}$ function for $x$ an open set $D \subset \mathfrak{R}^{n}$.

Consider the differential equation

$$
x^{\prime}=f(x) .
$$

Denote by $x\left(t, x_{0}\right)$ the solution to equation (6) such that $x\left(0, x_{0}\right)=x_{0}$.

Definition $1 A$ set $K$ is said to be absorbing in $D$ for equation (6) if $x\left(t, k_{1}\right) \subset K$ for each compact $K_{1} \subset D$ and $t$ sufficiently large.

The following two basic assumptions are made;

$\left(H_{1}\right)$ There exists a compact absorbing set $K \subset D$

$\left(H_{2}\right)$ Equation (6) has a unique equilibrium point $\overline{\mathrm{x}}$ in $D$.

The equilibrium point $\overline{\mathrm{x}}$ is said to be globally stable if it is locally stable and all trajectories in D converge to $\overline{\mathrm{x}}$. The assumptions $H_{1}$ and $H_{2}$ are satisfied if $\overline{\mathrm{x}}$ is globally stable in $D$. For epidemic models and many other biological models where the feasible region is a bounded cone, $H_{1}$ is equivalent to the uniform persistence of (6) (Butler \& Waltman, 1986).

The following global stability problem is formulated in Li \& Muldowney (1996).

Global stability problem: Under assumptions $H_{1}$ and $H_{2}$, find conditions on the vector field of equation (6) such that the local stability of $\overline{\mathrm{x}}$ implies its global stability in $D$.

System (6) is said to satisfy the Poincaré-Bendixson property if any non-empty compact omega set of equation (6) that contains no equilibria is a closed orbit. 
Any autonomous system (6) in the plane satisfies the Poincaré-Bendixson property (Hale,1969). In addition, a threedimensional competitive system satisfies the Poincaré-Bendixson property in a convex region (Hirsch,1990).

The following global stability result is proved in Li \& Muldowney (1995).

\section{Theorem 1 Assume that}

1. assumptions $\left(H_{1}\right)$ and $\left(H_{2}\right)$ hold;

2. $\bar{x}$ is locally asymptotically stable

3. System (6) satisfies the Poincaré- Bendixson property;

4. each periodic orbit of (6) in D is orbitally asymptotically stable.

Then the unique equilibrium $\overline{\mathrm{x}}$ is globally asymptotically stable in $D$. Assumption (3) is satisfied if $D$ is a convex region in $\mathfrak{R}^{3}$ and (6) is a competitive system in $D$.

The orbital stability of periodic solutions in $\mathfrak{R}^{n}(n \geq 2)$ can be verified using the following result of Muldowney (1990). Theorem 2 A periodic orbit $\Omega=\{p(t): 0 \leq t<\omega\}$ is orbitally asymptotically stable with asymptotic phase if the linear system

$$
z^{\prime}(t)=\frac{\partial f^{[2]}}{\partial x}(p(t)) z(t)
$$

is asymptotically stable where $\frac{\partial f^{[2]}}{\partial x}$ is the second additive compound matrix $\frac{\partial f}{\partial x}$ of $f$.

Definition 2 A matrix is stable if all it's eigenvalues have negative real parts.

Theorem 3 An $n \times n$ real matrix $A$ is stable if and only if $A^{[2]}$ is stable and $(-1)^{n} \operatorname{det}(A)>0$. (Li \& Wang,1998)

Using the theorems (1), (2), (3) above, the following result is used to establish the global stability of the endemic equilibrium point.

\section{Theorem 4 Assume that}

1. assumptions $\left(H_{1}\right)$ and $\left(H_{2}\right)$ hold;

2. system (6) satisfies a Poincaré-Bendixson property;

3. For each periodic solution $x=p(t)$ to (6) with $p(0) \in D$, system (6) is asymptotically stable;

4. $(-1)^{n} \operatorname{det}\left(\frac{\partial f}{\partial x}(\bar{x})\right)>0$

Then the unique equilibrium $\bar{x}$ is globally asymptotically stable in $D$.

This approach is used in order to establish the global stability of the endemic equilibrium point. System (3) is uniformly persistent, (see Proposition (3.3) in Li et al. (1999)), if there exists a constant $0<c<1$ such that any solutions $\left(s_{h}(t), i_{h}(t), i_{v}(t)\right)$ with $\left(s_{h}(0), i_{h}(0), i_{v}(0)\right) \in \dot{\Gamma}$ satisfies

$$
\min \left\{\lim _{t \rightarrow \infty} \inf s_{h}(t), \lim _{t \rightarrow \infty} \operatorname{infi}_{h}(t), \lim _{t \rightarrow \infty} \inf i_{v}(t)\right\}>c .
$$

The boundedness of $\Gamma$ and condition (8) imply that (3) has a compact absorbing set $K \subset \dot{\Gamma}$ [Butler \& Waltman,1986].

Definition 3 A differential equation (6) is said to be competitive in $D$ iffor some diagonal matrix $H=\operatorname{diag}\left(e_{1}, e_{2}, e_{3} \ldots . . . e_{n}\right)$, where each $e_{i}$ is either -1 or +1 , then $H\left(\frac{\partial f}{\partial x}\right) H$ has non-positive off diagonal elements for all $x \in D$. If $D$ is convex, the flow of a competitive system preserves for $t<0$, the partial ordering in $\mathfrak{R}^{n}$ defined by the orthant $K=\left\{\left(x_{1}, x_{2}, \ldots \ldots . x_{n}\right) \in\right.$ $\left.\mathfrak{R}^{n}: e_{i} x_{i} \geq 0\right\}$.

To show that system (3) is a competitive system in the convex region $\dot{\Gamma}$, consider a partial ordering defined by the orthant $\left\{\left(s_{h}, i_{h}, i_{v}\right) \in \mathfrak{R}^{n}: s_{h} \leq 0, i_{h} \geq 0, i_{v} \leq 0\right\}$ and choose

$$
H=\left(\begin{array}{ccc}
-1 & 0 & 0 \\
0 & 1 & 0 \\
0 & 0 & -1
\end{array}\right)
$$


and using (5), then it can easily be verified that $H J_{E_{3}} H$ has no non-positive diagonal elements for all $x \in D$. Thus, system (3) is competitive in $\omega$, with respect to the partial ordering defined by the orthant $\left\{\left(s_{h}, i_{h}, i_{v}\right) \in \mathfrak{R}^{n}: s_{h} \leq 0, i_{h} \geq 0, i_{v} \leq 0\right\}$. The competitiveness of system (3) in a convex region $\dot{\Gamma}$ implies that system (3) satisfies a Poincaré-Bendixson property and thus conditions (1) and (2) hold. Using the substitution $s_{h}=1-i_{h}$, system (3) can be re-written as

$$
\begin{aligned}
s_{h}^{\prime} & =(\alpha+\phi) s_{h} i_{h}-\beta_{1} m s_{h} i_{v}, \\
i_{h}^{\prime} & =\beta_{1} m s_{h} i_{v}-(\alpha+\phi) s_{h} i_{h}, \\
i_{v}^{\prime} & =\beta_{2} i_{h}\left(1-i_{v}\right)-g i_{v} .
\end{aligned}
$$

whose Jacobian matrix is

$$
J_{E_{3}}=\left(\begin{array}{ccc}
(\alpha+\phi) i_{h}-\beta_{1} m i_{v} & (\alpha+\phi) s_{h} & -\beta_{1} m s_{h} \\
\beta_{1} m i_{v}-(\alpha+\phi) i_{h} & -(\alpha+\phi) s_{h} & \beta_{1} m s_{h} \\
0 & \beta_{2}\left(1-i_{v}\right) & -\beta_{2} i_{h}-g
\end{array}\right) .
$$

The second compound matrix of the Jacobian matrix (11)

$$
J_{E_{3}}^{[2]}=\left(\begin{array}{ccc}
(\alpha+\phi)\left(i_{h}-s_{h}\right)-\beta_{1} m i_{v} & \beta_{1} m s_{h} & \beta_{1} m s_{h} \\
\beta_{2}\left(1-i_{v}\right) & (\alpha+\phi) i_{h}-\beta_{1} m i_{v}-\beta_{2} i_{h}-g & (\alpha+\phi) s_{h} \\
0 & \beta_{1} m i_{v}-(\alpha+\phi) i_{h} & -(\alpha+\phi) s_{h}-\beta_{2} i_{h}-g
\end{array}\right)
$$

And the second compound matrix of system (11) along a periodic solution $\left(s_{h}(t), i_{h}(t), i_{v}(t)\right)$ is

$$
\begin{aligned}
X^{\prime} & =\left[(\alpha+\phi)\left(i_{h}-s_{h}\right)-\beta_{1} m i_{v}\right] X+\beta_{1} m s_{h} Y+\beta_{1} m s_{h} Z, \\
Y^{\prime} & =\beta_{2}\left(1-i_{v}\right) X+\left[(\alpha+\phi) i_{h}-\beta_{1} m i_{v}-\beta_{2} i_{h}-g\right] Y+(\alpha+\phi) s_{h} Z, \\
Z^{\prime} & =\left[\beta_{1} m i_{v}-(\alpha+\phi) i_{h}\right] Y+\left[-(\alpha+\phi) s_{h}-\beta_{2} i_{h}-g\right] Z .
\end{aligned}
$$

To show that system (13) is asymptotically stable, consider the following Lyapunov function

$$
V\left(X, Y, Z ; s_{h}, i_{h}, i_{v}\right)=\sup \left[|X| \frac{i_{h}}{i_{v}}(|Y|+|Z|)\right] .
$$

Then, the orbit $\psi$ of the periodic solution $\left(s_{h}(t), i_{h}(t), i_{v}(t)\right)$ is at a positive distance from the boundary $\partial \Gamma$ by uniform persistence. Thus, there exists a constant $c_{1}>0$ such that

$$
V\left(X, Y, Z ; s_{h}, i_{h}, i_{v}\right) \geq c_{1} \sup \left\{|X|, \frac{i_{h}}{i_{v}}(|Y|+|Z|)\right\}
$$

for all $(X, Y, Z) \in \mathfrak{R}^{3}$ and $\left(s_{h}, i_{h}, i_{v}\right) \in \psi$. The left hand derivative of $V$ along a solution $(X(t), Y(t), Z(t))$ to system (13) and $\left(s_{h}, i_{h}, i_{v}\right)$ can be estimated as follows:

$$
\begin{gathered}
D_{+}|X(t)| \leq\left[(\alpha+\phi)\left(i_{h}-s_{h}\right)-\beta_{1} m i_{v}\right]|X(t)|+\beta_{1} m s_{h}|Y(t)|+\beta_{1} m s_{h}|Z(t)|, \\
=\left[(\alpha+\phi)\left(i_{h}-s_{h}\right)-\beta_{1} m i_{v}\right]|X(t)|+\beta_{1} m s_{h}(|X(t)|+|Z(t)|), \\
D_{+}|X(t)|=\left[(\alpha+\phi)\left(i_{h}-s_{h}\right)-\beta_{1} m i_{v}\right]|X(t)|+\frac{\beta_{1} m s_{h} i_{v} i_{h}}{i_{h}} \frac{i_{h}}{i_{v}}[|Y(t)|+|Z(t)|], \\
D_{+}|Y(t)| \leq \beta_{2}\left(1-i_{v}\right)|X(t)|+\left[(\alpha+\phi) i_{h}-\beta_{1} m i_{v}-\beta_{2} i_{h}-g\right]|Y(t)|+\left[(\alpha+\phi) s_{h}\right]|Z(t)|, \\
D_{+}|Z(t)| \leq\left[\beta_{1} m i_{v}-(\alpha+\phi) i_{h}\right]|Y(t)|-\left[(\alpha+\phi) s_{h}+\beta_{2} i_{h}+g\right]|Z(t)| .
\end{gathered}
$$

Now, from inequalities (17) and (18), the following is obtained

$$
D_{+}\left(|Y(t)|+D_{+}|Z(t)|\right) \leq \beta_{2}\left(1-i_{v}\right)|X(t)|-\left(\beta_{2} i_{h}+g\right)(|Y(t)|+|Z(t)|),
$$

such that

$$
D_{+} \frac{i_{h}}{i_{v}}(|Y(t)|+|Z(t)|) \leq \beta_{2} \frac{i_{h}}{i_{v}}\left(1-i_{v}\right)|X(t)|+\left(\frac{i_{h}^{\prime}}{i_{h}}-\frac{i_{v}^{\prime}}{i_{v}}-\beta_{2} i_{h}-g\right) \frac{i_{h}}{i_{v}}(|Y(t)|+|Z(t)|)
$$

Equations (16) and (20) lead to

$$
D_{+} V(t) \leq \max \left\{g_{1}(t), g_{2}(t)\right\} V(t)
$$


where

$$
\begin{array}{r}
g_{1}(t)=(\alpha+\phi)\left(i_{h}-s_{h}\right)-\beta_{1} m i_{v}+\frac{\beta_{1} m i_{v} s_{h}}{i_{h}}, \\
g_{2}(t)=\frac{\beta_{2} i_{h}\left(1-i_{v}\right)}{i_{v}}+\left(\frac{i_{h}^{\prime}}{i_{h}}-\frac{i_{v}^{\prime}}{i_{v}}-\beta_{2} i_{h}-g\right) .
\end{array}
$$

From system (3),

$$
\begin{aligned}
& i_{h}^{\prime}=\beta_{1} m s_{h} i_{v}-(\alpha+\phi) s_{h} i_{h}, \\
& \frac{\beta_{1} m s_{h} i_{v}}{i_{h}}=\frac{i_{h}^{\prime}}{i_{h}}+(\alpha+\phi) s_{h} .
\end{aligned}
$$

Substituting equation (24) into equation (22) and using the expressions for $i_{v}$ and $i_{h}$ at the endemic equilibrium point gives

$$
\begin{aligned}
g_{1}(t) & =\frac{i_{h}^{\prime}}{i_{h}}-\beta_{1} m i_{v}+(\alpha+\phi) i_{h}, \\
& =\frac{i_{h}^{\prime}}{i_{h}}-\beta_{1} m \frac{\left(R_{0}^{2}-1\right)}{R_{0}^{2}}+(\alpha+\phi)\left(R_{0}^{2}-1\right) \frac{g}{\beta_{2}}, \\
& =\frac{i_{h}^{\prime}}{i_{h}}-\beta_{1} m \frac{\left(R_{0}^{2}-1\right)}{R_{0}^{2}}+\frac{\beta_{1} \beta_{2} m}{R_{0}^{2}} \frac{\left(R_{0}^{2}-1\right)}{\beta_{2}}, \\
& =\frac{i_{h}^{\prime}}{i_{h}} .
\end{aligned}
$$

Similarly, from system (3),

$$
\begin{aligned}
& i_{v}^{\prime}=\beta_{2} i_{h}\left(1-i_{v}\right)-g i_{v}, \\
& \frac{\beta_{2} i_{h}\left(1-i_{v}\right)}{i_{v}}=\frac{i_{v}^{\prime}}{i_{v}}+g .
\end{aligned}
$$

Substituting equation (25) into equation (23) gives

$$
\begin{aligned}
g_{2}(t) & =\frac{i_{h}^{\prime}}{i_{h}}-\beta_{2} i_{h}, \\
& =\frac{i_{h}^{\prime}}{i_{h}}-g\left(R_{0}^{2}-1\right) .
\end{aligned}
$$

Thus,

$$
\begin{aligned}
D_{+} V(t) & \leq \max \left\{g_{1}(t), g_{2}(t)\right\} V(t) \\
& \leq \frac{i_{h}^{\prime}}{i_{h}}
\end{aligned}
$$

Therefore,

$$
\begin{aligned}
\int_{0}^{\omega} D_{+} V(t) & \leq\left.\log i_{h}(t)\right|_{0} ^{\omega} \\
& =0
\end{aligned}
$$

since $i_{h}(t)$ is periodic of minimal period $\omega$. This relation together with (21) above imply that, $V(t) \longrightarrow 0$ as $t \longrightarrow \infty$ and in turn that $(X(t), Y(t), Z(t)) \longrightarrow 0$ as $t \longrightarrow \infty$ by (15). As a result, the second compound system (13) is asymptotically stable if the minimal period $\omega>0$. The same estimate also holds when $\omega \longrightarrow 0$. This verifies condition (3).

It now remains to show whether (5) is stable. Recall $p=\frac{g}{\beta_{2}}\left(R_{0}^{2}-1\right)=i_{h}$ such that $s_{h}=1-p$. Then, $J_{E_{3}}$ can be re-written as

$$
J_{E_{3}}=\left(\begin{array}{ccc}
-\alpha(1-p) & \phi(1-p) & -\beta_{1} m(1-p) \\
\phi p & -\alpha(1-p)-\phi(1-2 p) & \beta_{1} m(1-p) \\
0 & \frac{\beta_{2}}{R_{0}^{2}} & -g R_{0}^{2}
\end{array}\right)
$$


Now,

$$
\begin{aligned}
& \operatorname{det}\left(J_{\left(E_{3}\right)}\right)= \\
& =-\alpha(1-p)\left\{[\alpha(1-p)+\phi(1-2 p)]\left[g R_{0}^{2}\right]-\beta_{1} m(1-p) \frac{\beta_{2}}{R_{0}^{2}}\right\}+\phi(1-p)\left(g R_{0}^{2} p \phi\right)-\beta_{1} m(1-p) \phi p\left(\frac{\beta_{2}}{R_{0}^{2}}\right), \\
& =-\alpha(1-p)\left\{g R_{0}^{2}[\alpha(1-p)+\phi(1-p)-\phi p]-g(\alpha+\phi)(1-p)\right\}+\phi^{2} p(1-p) g R_{0}^{2}-g p \phi(\alpha+\phi)(1-p), \\
& =-\alpha(1-p) g R_{0}^{2}[(\alpha+\phi)(1-p)-p \phi]+g \alpha(\alpha+\phi)(1-p)^{2}+\phi^{2} p g(1-p) R_{0}^{2}-g p \phi(\alpha+\phi)(1-p), \\
& =g(1-p) R_{0}^{2}\left[-\alpha(\alpha+\phi)(1-p)+\alpha p \phi+p \phi^{2}\right]+g(\alpha+\phi)(1-p)[\alpha(1-p)-p \phi], \\
& =g(1-p)(\alpha+\phi) R_{0}^{2}[-\alpha(1-p)+p \phi]+g(\alpha+\phi)(1-p)[\alpha-p(\alpha+\phi)], \\
& =g(1-p)(\alpha+\phi) R_{0}^{2}[p(\alpha+\phi)]-g(\alpha+\phi)(1-p)[p(\alpha+\phi)-\alpha], \\
& =g(1-p)(\alpha+\phi)[p(\alpha+\phi)-\alpha]\left\{R_{0}^{2}-1\right\} .
\end{aligned}
$$

Since $\alpha>p(\alpha+\phi)$, then it is clear that determinant $J_{E_{3}}<0$ if $R_{0}>1$ and therefore $J_{E_{3}}$ is stable. This satisfies condition (4) above. Since all conditions of Theorem 4 have been verified, the endemic equilibrium point $E_{3}$ is globally asymptotically stable if $R_{0}>1$.
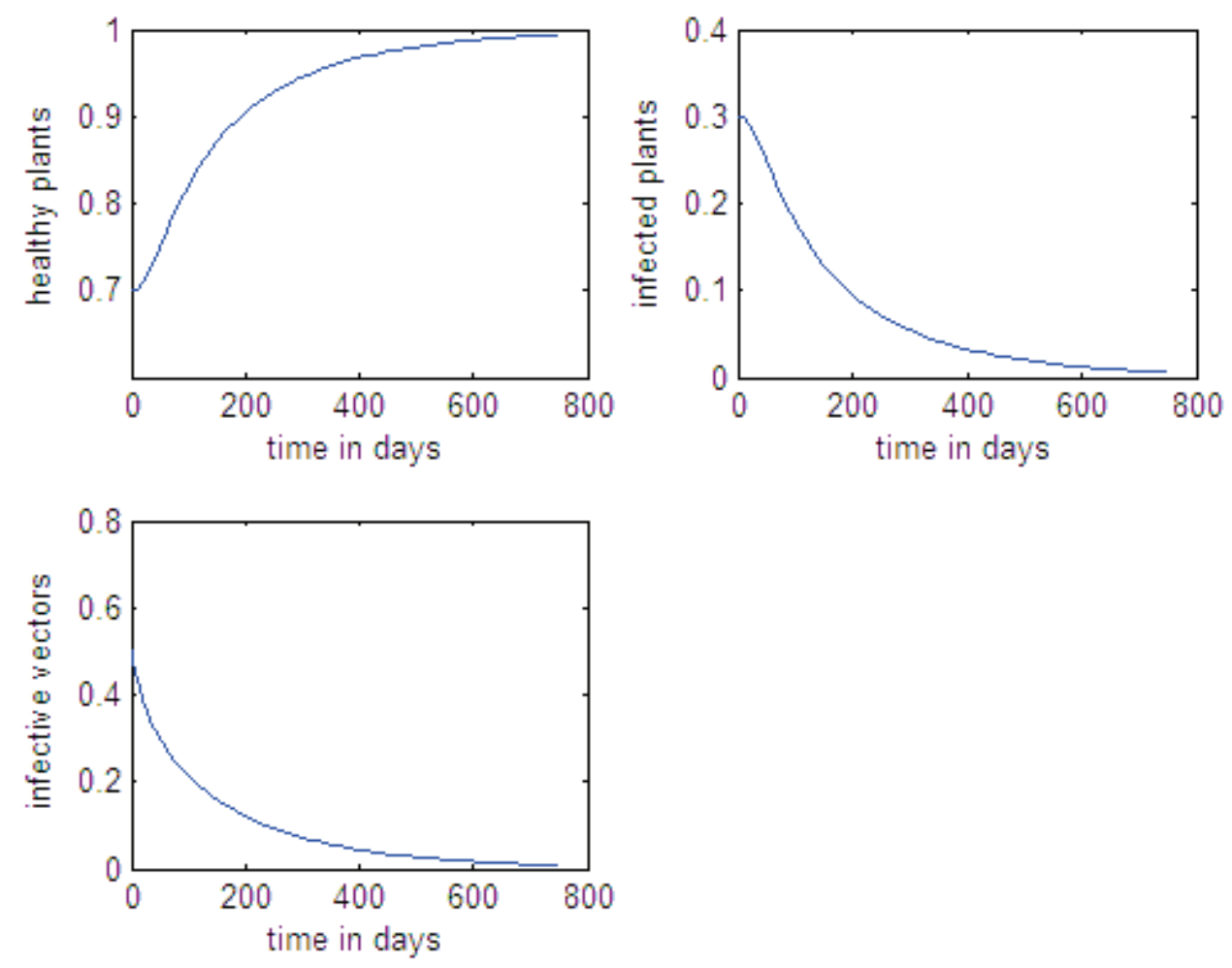

Figure 1. Variation of plant and vector proportions at the disease free equilibrium with initial values $s_{h}=0.7, i_{h}=0.3, i_{v}=$ 0.5 and parameters $\lambda_{1}=0.001, \mu=0.0056, r=0.0105, d=0.0167, g=0.02, \beta_{1}=\beta_{2}=0.021, m=1$

\section{Numerical Simulation}

In this section, we provide a numerical analysis of the model to support the theoretical analysis carried out in Section 2. A set of estimated parameter values are obtained from literature. The model is simulated using Matlab ode 45 solver. We simulate the model both in the absence and presence of the disease, and without control methods in place.

\section{Discussion}

In the paper, a mathematical model for the vector transmission and control of banana Xanthomonas wilt incorporating roguing of infected plants and replanting using healthy or disease free suckers was formulated. The steady states were obtained and their stability established. The basic reproduction number was determined using the next generation matrix 

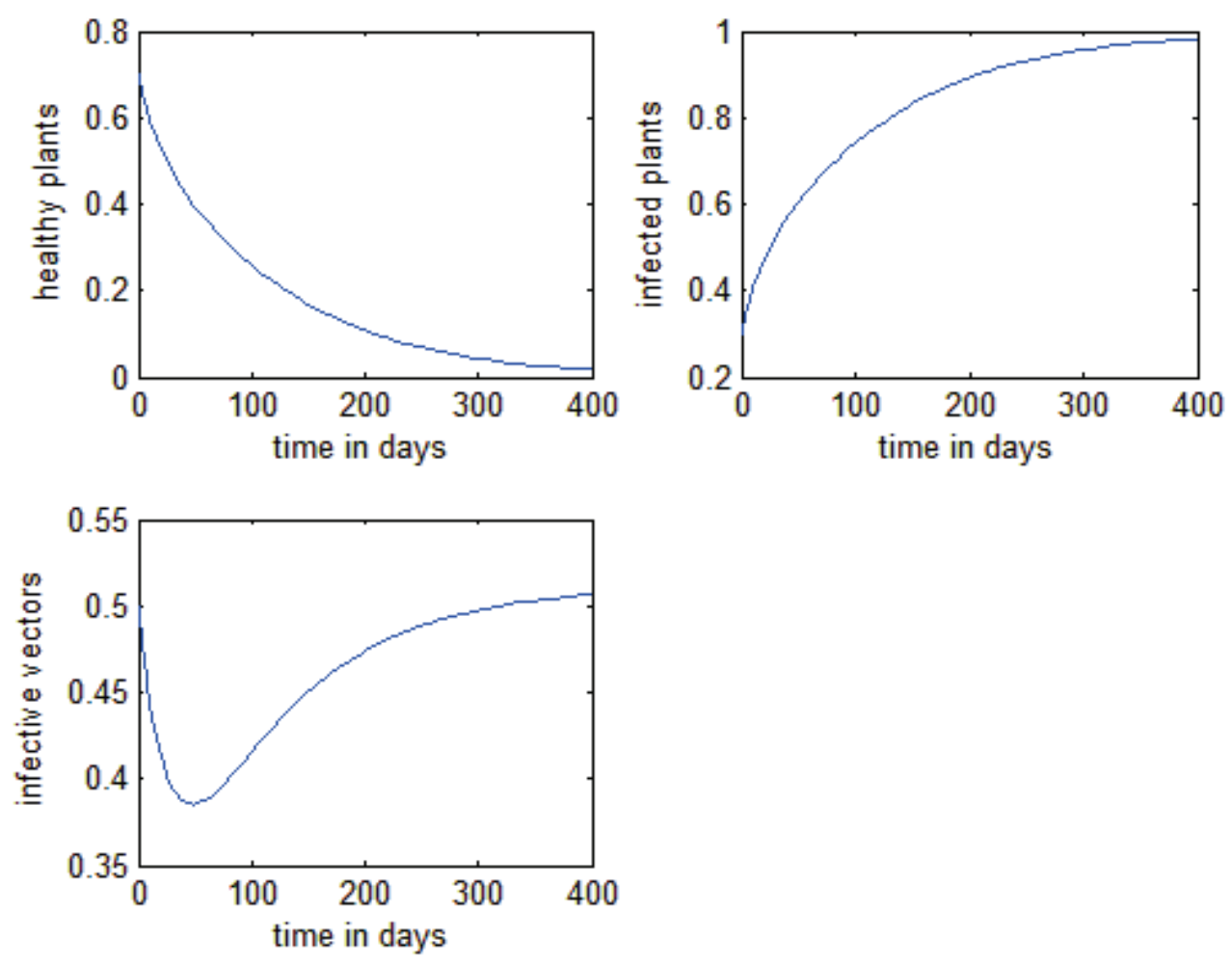

Figure 2. Variation of plant and vector proportions with neither roguing of infected plants nor replanting using healthy suckers
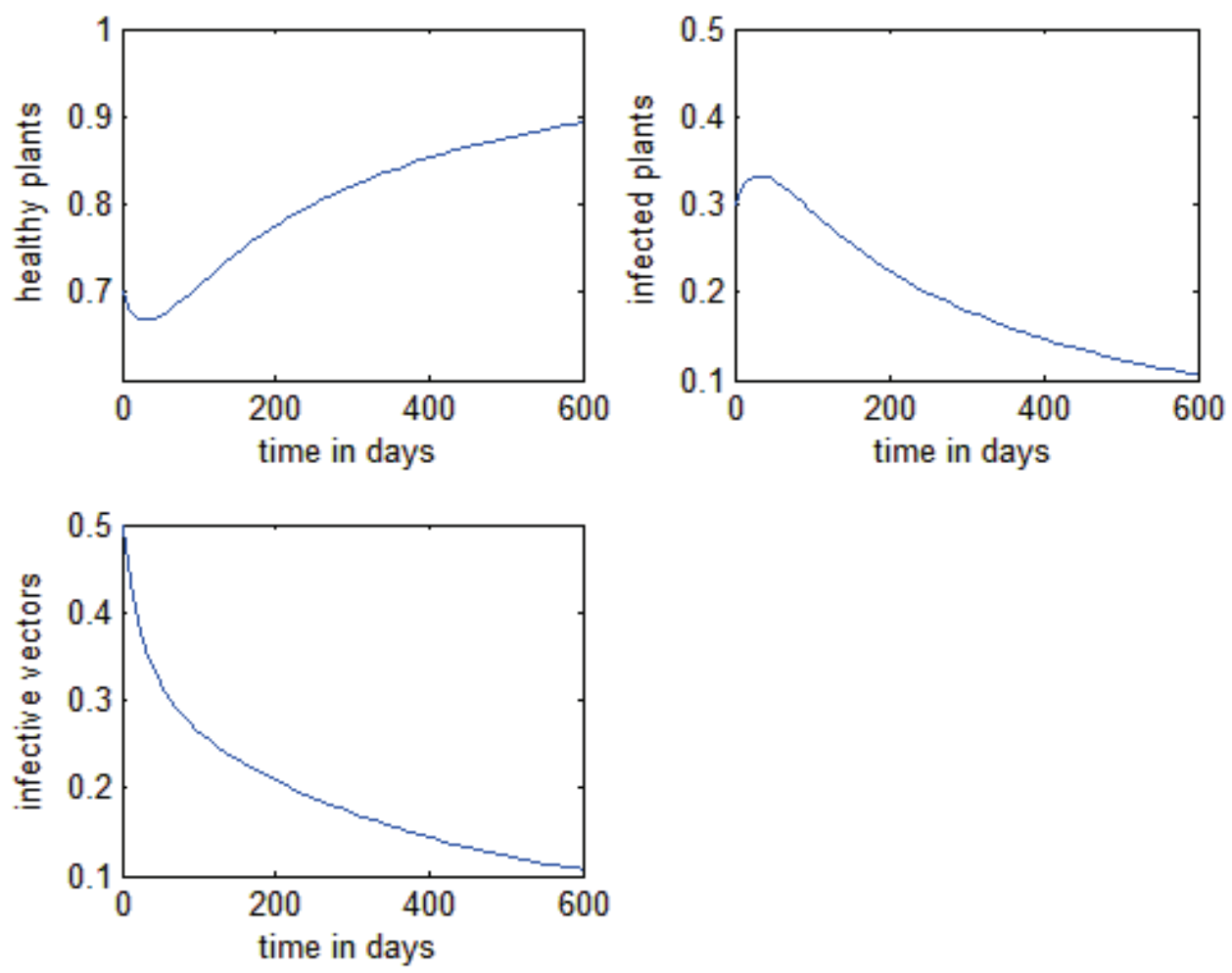

Figure 3. Variation of plant and vector proportions at the endemic equilibrium. 
and its implications for disease management analyzed. It was revealed that $R_{0}$ is a combination of two basic reproduction numbers namely $R_{0}^{\prime}=\frac{\beta_{1} m}{\lambda_{1}+2 r+d-\mu}$ for banana plants, that is, the number of secondary infected banana plants arising from one infected banana plant introduced in a plantation of completely healthy plants and $R_{0}^{\prime \prime}=\frac{\beta_{2}}{g}$ the basic reproduction number for the vectors, that is, the average number of secondary infective vectors that arise out of one infective vector introduced into a population of completely susceptible or pathogen-free vectors. When $R_{0}^{\prime}>1$ the disease grows in the plant hosts and when $R_{0}^{\prime \prime}>1$ then pathogen spreads in the vector population. The two conditions enable the net reproduction number $R_{0}$ to be greater than unity. It was also observed that $R_{0}$ is directly proportional to the transmission or contact rates $\beta_{1}, \beta_{2}$ and the vector-plant ratio, $m$. $R_{0}$ is inversely proportional to vector immigration/emigration rate, $g$, the sucker emergence rate, $\lambda_{1}$, the death rate $d$ and roguing rate $r$ of the infected plants.

Disease management is concerned with lowering the basic reproduction number to a value less than unity. In this case, reduction of the basic reproduction number, $R_{0}$ to a value less than unity involves reducing the contact rates $\beta_{1}, \beta_{2}$ between the banana plants and the vectors and reducing the vector-plant ratio, $m$. Contact between the vector and the banana plant is made at the male buds and therefore removal of the male buds is key. Removal of male buds also ensures that the number of insect vectors visiting the plant is also reduced thereby reducing the vector-plant ratio, $\mathrm{m}$. Reducing $R_{0}$ to a value less than unity can also be achieved by increasing the roguing rate, $r$, of infected plants, increasing the emigration rate $g$ of vectors, increasing the replanting rate of healthy suckers $\lambda_{1}$ and increasing the disease-induced death rate, $d$, of infected plants.

Increasing the roguing rate, $r$, of infected plants and the replanting rate using healthy suckers are crucial in managing the outbreak of banana Xanthomonas wilt. At low contact rates and high roguing rate, the disease can be averted as shown in Figure 1 while in absence of roguing and replanting, the entire healthy plant population is wiped out in finite time as shown in Figure 2. This calls for regular inspection of the banana plantation and prompt roguing of identified diseased plants. It should be noted that where this practice has been strictly applied in Uganda, the disease has been contained. The challenge with replanting using healthy or disease free suckers is absence of certified healthy suckers for replanting. No formal supply of disease free planting materials is available in Uganda within the areas currently affected by banana Xanthomonas wilt and consequently, most planting materials are sourced locally by farmers from their own or neighbors fields. Planting materials sourced in this way may appear to be healthy when in fact they are already diseased. This is suspected to be the major method of disease introduction to new areas.

Lastly, increasing the death rate of diseased plants ensures reduction in the inoculum load present. There is need to destroy the infected plants as soon as they are identified thereby killing the bacterium in the process. The greatest challenge is what happens to the infected plants removed by roguing? Unless they are completely destroyed especially by burning them, they can act as a source of the bacterium themselves. In Uganda, farmers have been encouraged to bury the plants removed by roguing but it is feared this may introduce the bacterium in the soil. In the end they are just left lying in plantation which poses a great danger. This calls for more research.

\section{Acknowledgements}

Eliab Horub Kweyunga is grateful for the financial support from the Belgium Technical Cooperation (BTC) Ref No: L09UGA/108 in the production of this manuscript. The authors thank the reviewers for their helpful comments.

\section{References}

Anderson, R. M., \& May, R. M. (1991). Infectious Diseases of Humans. Oxford University Press, Oxford.

Buregyeya, H., Kubiriba, J., ..., \& Kityo, R. (2014). Role of birds and bats in long distance transmission of banana bacterial wilt in Uganda. International Journal of Innovations and Research, 2(4),636-640.

Butler, G. J., \& Waltman, P. (1986). Persistence in dynamical systems. Proceedings of American Mathematical Society, 96, 425-430. https://doi.org/10.1090/S0002-9939-1986-0822433-4

Chan, M. S., \& Jeger, M. J. (1994). An analytical model of plant virus disease dynamics with roguing and replanting. Journal of Applied Ecology, 31, 413-427. https://doi.org/10.2307/2404439

Eden-Green, S. J. (2004). How can the advance of banana Xanthomonas campestris pv. musacearum wilt be halted? Infomusa, 13(2), 38-41.

Fishman, S., \& Marcus, R. (1984). A model for spread of plant disease with periodic removals. Journal of Mathematical Biology, 21, 149-158. https://doi.org/10.1007/BF00277667

Fishman, S., Marcus, R., ...,\& Zohar, M. (1983). Epidemiological and economic models for spread and control of citrus tristeza virus disease. Phytoparasitica, 11, 39-49.

https://doi.org/10.1007/BF02980710 
Gibson, R. W., Aritua, V., ..., \& Kayongo, J. (2004). Control strategies for Sweet potato virus disease in Africa. Virus Research, 100, 115-122. https://doi.org/10.1016/j.virusres.2003.12.023

Hale, J. K. (1969). Ordinary Differential Equations, John Wiley and Sons, New York.

Hirsch, M. W. (1990). Systems of differential equations which are competitive or cooperative 4: Structural stability in three dimensions, SIAM. Journal of Mathematical Analysis, 21, 1225-1234. https://doi.org/10.1137/0521067

Holt, J., Jeger, M. J., ...,\& Otin-Nape, G. W. (1997). An epidemiological model incorporating vector population dynamics applied to African Cassava Mosaic disease. Journal of Applied Ecology, 34, 793-806. https://doi.org/10.2307/2404924

Holt, J., Colvin, J ., \& Muniyappa, R. (1999). Identifying control strategies for tomato leaf curl virus disease using an epidemiological model. Journal of Applied Ecology, 36(5), 625-633. https://doi.org/10.1046/j.1365-2664.1999.00432.x

Jeger, M. J., Holt, J., ..., \& Madden, L. V. (2004). Epidemiology of insect transmitted plant viruses: modelling dynamics and control interventions.Physiological Entomology, 29, 291-304. https://doi.org/10.1111/j.0307-6962.2004.00394.x

Jogo, W., Karamura, E., ..., \& Rietveld. A. (2013). Determinants of farm-level adoption of cultural practices for banana Xanthomonas wilt control in Uganda. Journal of Agricultural Science, 5(7), 70-79. http://dx.doi.org/10.5539/jas.v5n7p70

Kubiriba, J., Muthomi, J., ..., \& Opio, F. (2014).Strategies for rehabilitation of banana fields infested with Xanthomonas campestris pv. musacearum. Journal of Crop Protection, 3(1), 21-29.

Kubiriba, J., \& Tushemereirwe, W. K. (2014). Approaches for the control of banana Xanthomonas wilt in East and Central Africa. African Journal of Plant Science, 8(8), 398-404. 10.5897/AJPS2013.1106

LaSalle, J. P. (1976). The stability of dynamical systems, Regional Conference Series in Applied Mathematics, SIAM, Philadelphia.

Li, M. Y, \& Muldowney, J. S. (1996). A geometric approach to global- stability problems, SIAM. Journal of Mathematical Analysis, 27, 1070-1083.

Li, Y., \& Muldowney, J. S. (1995). Global stability of the SEIR model in Epidemiology. Mathematical Biosciences, 125, 155-164. https://doi.org/10.1016/0025-5564(95)92756-5

Li, M. Y., Graef, J. R., ..., \& Karsai, J. (1999). Global dynamics of a SEIR model with a varying total population size. Mathematical Biosciences, 160, 191-213. https://doi.org/10.1016/S0025-5564(99)00030-9

Li, M. Y., \& Wang, L.(1998). A criterion for stability of matrices. Journal of Mathematical Analysis and Applications, 225, 249-264. https://doi.org/10.1006/jmaa.1998.6020

Madden, L. V., Jeger, M. J., \& van den Bosch, F. (2000). A theoretical assessment of the effects of vector-virus transmission mechanism on plant virus disease epidemics. Phytopathology, 90, 576-594.

https://doi.org/10.1094/PHYTO.2000.90.6.576

Muldowney, J. S. (1990). Compound matrices and differential equations. Rocky Mountains Journal of Mathematics, 20 , 857-872. https://doi.org/10.1216/rmjm/1181073047

Nakakawa, J., Mugisha, J. Y. T., ...,\& Karamura, E. (2016). A mathematical model for the dynamics of banana $X$ anthomonas wilt with vertical transmission and inflorescence infection. Journal of Biological Systems, $24(1), 47$. https://doi.org/10.1142/S021833901650008X

Nannyonga, B., Luboobi, L. S., ..., \& Jablonska-Sabuka, M. (2015). Using contaminated tools fuels outbreaks of banana Xanthomonas wilt: An optimal control study within plantations using RungeKutta fourth-order algorithms. International Journal of Biomathematics, 8(5), 20 pages. https://doi.org/10.1142/S1793524515500655

Rutikanga, A., Tusiime, G., ..., \& Blomme, G. (2016). Variation in nectar volume and sugar content in male flowers of Musa cultivars grown in Rwanda and their non-effect on the numbers of visiting key diurnal insect vectors of banana Xanthomonas wilt. African Journal of Agricultural Research, 11(8), 607-623. http://hdl.handle.net/10568/74521

Tang, S. Y., Xiao, Y. N., \& Cheke, R. A. (2010). Dynamical analysis of plant disease models with cultural control strategies and economic thresholds. Mathematics and Computers in Simulation, 80(5), 894-921.

Tinzaara, W., Karamura, E. B., ..., \& Ssekiwoko, F. (2016). The banana Xanthomonas wilt epidemic in east and central Africa: current research and development efforts. Acta Horticulturae, 1114, 267-274. 
https://doi.org/10.17660/ActaHortic.2016.1114.36

Tinzaara, W., Gold, C. S., ..., \& Eden-Green, S. J. (2006). Role of insects in the transmission of banana bacterial wilt. African Crop Science Journal, 14(2), 105-110.

Tripathi, L., Tripathi, J. N., \& Tushemereirwe, W. K. (2008). Rapid and efficient production of transgenic East African Highland Banana using intercalary meristematic tissues. African Journal of Biotechnology, 7, 1438-1445.

Tushemereirwe, W. K., Kangire, W., ..., \& Smith, J. J. (2004). First report of Xanthomonas campestris pv.musacearum on banana in Uganda. Plant Pathology, 53, 802. https://doi.org/10.1111/j.1365-3059.2004.01090.x

van den Driessche, P., \& Watmough, J. (2002). Reproduction numbers and the sub-threshold endemic equilibria for compartmental models of disease transmission. Mathematical Biosciences, 180 (2), 29-48. https://doi.org/10.1016/S00255564(02)00108-6

Zhonghua, Z., \& Yaohong, S. (2014). Stability and sensitivity analysis of a plant disease model with continuous cultural control strategy. Journal of Applied Mathematics, 2014, 15 pages. http://dx.doi.org/10.1155/2014/207959

\section{Copyrights}

Copyright for this article is retained by the author(s), with first publication rights granted to the journal.

This is an open-access article distributed under the terms and conditions of the Creative Commons Attribution license (http://creativecommons.org/licenses/by/4.0/). 